\title{
Porous Carbon Materials in Biomedical Applications
}

\author{
Charitha Thambiliyagodage* Shanitha Mirihana and Himasha Gunathilaka \\ Faculty of Humanities and Sciences, Sri Lanka Institute of Information Technology, Sri Lanka \\ All authors equally contributed
}

*Corresponding author: Charitha Thambiliyagodage, Faculty of Humanities and Sciences, Sri Lanka Institute of Information

Technology, New Kandy Road, Malabe, Sri Lanka

\section{ARTICLE INFO}

Received: 幽 November 07, 2019

Published: 慧 November 12, 2019

Citation: Charitha Thambiliyagodage, Shanitha Mirihana, Himasha Gunathilaka. Porous Carbon Materials in Biomedical Applications. Biomed J Sci \& Tech Res 22(4)-2019. BJSTR. MS.ID.003798.

\begin{abstract}
Recent advances in carbon based porous materials in biomedical science and technology have been discussed. Biomedical applications like drug delivery, tissue engineering, biomedicine, gene transfection, photothermal and photo-dynamic theory of porous carbon materials such as mesoporous carbon materials, carbon nanotubes, activated carbon, graphene and fullerenes have been briefed. Finally, future biomedical applications of new porous carbon materials including metal organic frameworks, carbon dots have been introduced in this mini review.
\end{abstract}

Keywords: Mesoporous; Carbon Nanotubes; Activated Carbon; Fullerenes; Graphene; Delivery; Medicine

Abbreviations: PCM: Porous Carbon Materials; AC: Activated Carbon

\section{Introduction}

Porous carbon materials (PCM) can be classified depending on the size of the pores as microporous $<2 \mathrm{~nm}$, mesoporous $2-50 \mathrm{~nm}$ and macroporous $>50 \mathrm{~nm}$. PCM could contain only one type of porous system or can be a product of combination of two types or all three types of pores. When such combination of porous systems are present as an interconnected porous system such materials are called as hierarchically porous materials. Further the present single porous system or combination of porous systems could be ordered or disordered in shape, size and location. PCM can be synthesized mainly in two different ways, nanocasting where a hard template is used to build up the carbon structure and direct synthesis where a soft template is used. Hard templates used for nanocasting method are mainly zeolite and silica. Such hard template materials are embedded in the carbon precursor. Carbonization followed by the removal of template generates the porous structure. Zeolite template produces microporous carbon and silica template produces either mesoporous or macroporous or such resulting PCM can have all three porous systems as well [1]. Direct synthesis mainly involves the synthesis of PCM by sol-gel synthesis where a soft template, basically a polymer/surfactant along with the carbon precursor and a polymerization agent is used to generate the porous structure. In addition to such PCM synthesized by the above-mentioned methods, PCM generated by naturally available materials such as coconut husk, paddy husk, tea waste etc. are also available as low cost materials. PCM are promising candidates in the discipline of material science due to their high performance in extensive applications. These materials have received great attention due to their unique and distinctive physiochemical and biological properties including large surface area and large pore volume with adjustable pore structure, presence of favorable functional groups including $\pi-\pi$ stacking and a easily modifiable surface, high heat conversion capacity, unique optical properties, high biocompatibility, high chemical inertness, and high mechanical stability $[2,3]$. PCM are used in many applications including but not limited to supercapacitor applications and electrochemical applications [4], supports in catalysis [5], adsorbents to remove pollutants etc. [6]. This mini review summarizes the biomedical applications of such PCM.

\section{Mesoporous Carbon Materials}

Liu et al. [7] reported that the $\mathrm{Eu}^{3+} / \mathrm{Gd}^{3+}$ - EDTA-doped hollow mesoporous carbon was an effective drug delivery system with high loading capacity and sustained release behavior of the model drug carvedilol compared to the commercially available drug [7]. They have noticed that the dissolution rate decelerated with increasing mesoporous shell. Further, according to the findings by Wan et al. [8] hyaluronic functionalized mesoporous carbon spheres loaded 
with doxorubicin and verapamil has significantly increase the cell cytotoxicity and cell apoptosis and therefore has exhibited high anticancer effects with low systemic toxicity. Hence the reported mesoporous carbon spheres are effective drug carriers for efficient combination cancer therapy [8]. Zhang et al. [9] also reported mesoporous carbon nanospheres capped with $\mathrm{CuS}$ nanoparticles as an effective drug carrier using doxorubicin as the drug and due to the near-infrared absorption and photothermal conversion ability of CuS nanoparticles, generating heat could kill the tumor cells [9].

\section{Carbon Nanotubes}

Serag et al. [10] reported endosomes escaping multiwall carbon nanotube based nano transporters for plant cells which could be used to deliver molecular cargos into specific compartments of the cell [10]. Carbon nanotubes-based biosensors can overcome some disadvantages associated with the conventional electrochemical biosensors such as poor sensitivity and stability, large response time, low reproducibility, etc. [11]. Liu et al. [12] have found that the nanocomposite film which contains multi-walled carbon nanotubes, chitosan, poly(amidoamine) along with the incorporation of DNA, modified $\mathrm{Au}$ electrode exhibited electro-catalytic activity toward dopamine and uric acid oxidation under coexistence of ascorbic acid and could separate oxidation peaks of those compounds into three-well defined peaks facilitating the determination of dopamine and uric acid in the presence of large amounts of ascorbic acid [12]. Xing et al. [13] reported Lentinan modified multi walled carbon nanotubes could enter the dendritic cells and carry large amounts of antigen being an effective intracellular antigen depot and they can potentiate cellular and humoral immunity and constitute a potential vaccine delivery system for therapeutic purposes [13].

\section{Activated Carbon}

Activated carbon (AC) is utilized in fabrication of electrodes used in biosensors where the high surface area allows immobilization of enzymes required for sensing compound of interest and promoting the electron transfer between the electrode and substrate [14]. Biosensors fabricated mainly with reduced graphene oxideactivated carbon composites decorated with Pt nanoparticles exhibit good response to glucose with high sensitivity in a short response time of about $4 \mathrm{~s}$ and a lower detection limit of $2 \mu \mathrm{M}$ suggesting them to be used in urine glucose sensing applications [15]. AC has also been used in fabricating a biosensor designed to quantify triglycerides in human serum samples where the results obtained were on par with industrial standards [16]. Further, a biosensor fabricated with the aid of AC for quantification of Dopamine is reported to have minimized the interference by ascorbic acid in the assay and outperformed previously designed dopamine biosensors in sensitivity [17]. Magnetically targeted drug delivery is an area of research that realizes the potential of AC in adsorption and desorption of drugs like Theophylline to deliver and retain in an area of interest providing prolonged therapy [18]. Widely recognised for its adsorption capacity, activated carbon is being administered in treating cases of acute poisoning as either a single dose or multiple doses. A single dose of AC is generally employed in gastrointestinal decontamination upon ingestion of toxic substances which are known to be adsorbed to AC [19].

\section{Fullerenes}

Foley et al. [20] demonstrated through fluorescence microscopy and radioactive labelling that a water-soluble fullerene derivative $\mathrm{C}_{61}(\mathrm{COOH})_{2}$ is able to cross the cell membrane and preferentially bind to the mitochondria. This resembles the structural analogy between the fullerene cage and that of the clathrin-coated vesicles, which therefore can be used in organelle targeted drug delivery [20]. Fullerene is a spherical carbon molecule with strong radical activity and such theory has been used by Inui et al. [21] to find out the clinical effectiveness of fullerene gel in treating acne vulgaris. In this study, an open trial using a fullerene gel twice a day; at 4 and 8 weeks, showed a decrease in the mean number of inflammatory lesions [21]. Gharbi et al. [22] studied the free-radical scavenging properties of fullerene using acute carbon tetrachloride intoxication in rats which is a classical model for studying free-radical-mediated liver injury. This proved that aqueous fullerene suspension could act as a powerful liver-protective agent [22].

\section{Graphene}

Chowdhury et al. [23] reported the use of oxidized graphene nanoribbons as a delivery system to deliver anti-tumor drug Lucanthone into the cells reducing nonspecific cytotoxicity to the surrounding healthy tissues [23]. Zhang et al. [24] reported that the $\mathrm{BaGdF}_{5}$ nanoparticles attached graphene oxide nanosheets shows low cytotoxicity, positive magnetic resonance contrast effect enabling magnetic resonance and X-ray computed tomography imaging of tumor cells and could be used in photo thermal therapies [24]. Though how much graphene-based materials are promising candidates for biomedical applications, generally these materials show different degrees of toxicity limiting their use in such applications [25].

\section{Future Materials}

Metal organic framework derived nano parous carbon materials, and carbon quantum dots are potential candidates that could be modified and developed for biomedical applications in the future.

\section{Conclusion}

Various PCM have been developed and used in the biotechnological applications especially in biomedical applications. New candidates for such applications should also be developed that may withstand the disadvantages of the existing materials.

\section{References}

1. Lee J, Kim J, Hyeon T (2006) Recent progress in the synthesis of porous carbon materials. Advanced Materials 18(16): 2073-2094.

2. Zhao Q, Lin Y, Han N, Li X, Geng H, et al. (2017) Mesoporous carbon nanomaterials in drug delivery and biomedical application. Drug Delivery 24(1): 94-107. 
3. Chen Y, Shi J (2015) Mesoporous carbon biomaterials. Sci China Mater 58(3): 241-257.

4. Vix Guterl C, Frackowiak Elzbieta, Jurewicz Krzysztof, Friebe Marcin, Parmentier Julien, et al. (2005) Electrochemical energy storage in ordered porous carbon materials. Carbon N Y 43(6): 1293-1302.

5. Thambiliyagodage C, Hakat YG, Bakker M (2016) One Pot Synthesis of Carbon/Ni Nanoparticle Monolithic Composites by Nanocasting and Their Catalytic Activity for 4-Nitrophenol Reduction. Curr Catal 5(2): 135-146.

6. Thambiliyagodage CJ, Cooray VY, Perera IN, Wijesekera RD (2020) EcoFriendly Porous Carbon Materials for Wastewater Treatment pp. 252260.

7. Liu J (2016) $\mathrm{A} \mathrm{Eu}^{3+} / \mathrm{Gd}^{3+}$-EDTA-doped structurally controllable hollow mesoporous carbon for improving the oral bioavailability of insoluble drugs and in vivo tracing. Nanotechnology 27.

8. Wan L, Jiao J, Cui Y, Guo J, Han N, et al. (2016) Hyaluronic acid modified mesoporous carbon nanoparticles for targeted drug delivery to CD44overexpressing cancer cells. Nanotechnology 27(13):135102.

9. Zhang L, Li Y, Jin Z, Chan KM, Yu JC (2015) Mesoporous carbon/CuS nanocomposites for $\mathrm{pH}$-dependent drug delivery and near-infrared chemo-photothermal therapy. RSC Adv 5: 93226-93233.

10. Serag MF, Kaji N, Gaillard C, Okamoto Y, Terasaka K, et al. (2011) Trafficking and subcellular localization of multiwalled carbon nanotubes in plant cells. ACS Nano 5(1): 493-499.

11. Balasubramanian K, Burghard M (2006) Biosensors based on carbon nanotubes. Analytical and Bioanalytical Chemistry 385(3): 452-468.

12. Liu X, Yinghua Peng, Xiangjin Qu, Shiyun Ai (2011) Multi-walled carbon nanotube-chitosan/poly(amidoamine)/DNA nanocomposite modified gold electrode for determination of dopamine and uric acid under coexistence of ascorbic acid. J Electroanal Chem 654(1-2): 72-78.

13. Xing J, Zhenguang Liu, Yifan Huang, Tao Qin, Ruonan Bo, et al. (2016) Lentinan-Modified Carbon Nanotubes as an Antigen Delivery System Modulate Immune Response in Vitro and in Vivo. ACS Appl Mater Interfaces 8(30): 19276-19283.

14. Pinyou P, Blay V, Muresan LM, Noguer T (2019) Enzyme-modified electrodes for biosensors and biofuel cells. Mater. Horizons 6: 13361358.

ISSN: 2574-1241

DOI: 10.26717/BJSTR.2019.22.003798

Charitha Thambiliyagodage. Biomed J Sci \& Tech Res

This work is licensed under Creative

Commons Attribution 4.0 License

Submission Link: https://biomedres.us/submit-manuscript.php
15. Hossain MF, Park JY (2016) Plain to point network reduced graphene oxide-activated carbon composites decorated with platinum nanoparticles for urine glucose detection. Sci Rep 6: 21009.

16. Ömer Munzuroglu (1999) An Investigation on the Interaction of Plant Growth Substances and the Inhibitory Effects of Cigarette Smoke on Germination. Turkish J Biol 23: 115-126.

17. Rahman SF, Kyoungseon Min, Seok Hwan Park, Jae Hee Park, Jin Cheol Yoo, et al. (2016) Selective determination of dopamine with an amperometric biosensor using electrochemically pretreated and activated carbon/ tyrosinase/Nafion $®$-modified glassy carbon electrode. Biotechnol Bioprocess Eng 21(5): 627-633.

18. Ramanujan RV, Purushotham S, Chia MH (2007) Processing and characterization of activated carbon coated magnetic particles for biomedical applications. Mater Sci Eng C 27(4): 659-664.

19. Pfab R (2017) Single dose activated charcoal for gut decontamination: Application by medical non-professionals -a prospective study on availability and practicability. Toxicol Reports 4: 49-54.

20. Foley S, Crowley C, Smaihi M, Bonfils C, Erlanger BF, et al. (2002) Cellular localisation of a water-soluble fullerene derivative. Biochem Biophys Res Commun 294(1): 116-119.

21. Inui S, Aoshima H, Nishiyama A, Itami S (2011) Improvement of acne vulgaris by topical fullerene application: Unique impact on skin care. Nanomedicine Nanotechnology Biol Med 7(2): 238-241.

22. Gharbi N, Pressac M, Hadchouel M, Szwarc H, Wilson SR, et al. (2005) [60] Fullerene is a powerful antioxidant in vivo with no acute or subacute toxicity. Nano Lett 5(12): 2578-2585.

23. Chowdhury SM, Surhland C, Sanchez Z, Chaudhary P, Suresh Kumar MA, et al. (2015) Graphene nanoribbons as a drug delivery agent for lucanthone mediated therapy of glioblastoma multiforme. Nanomedicine Nanotechnology Biol Med 11(1): 109-118.

24.Zhang H, Wu H, Wang J, Yang Y, Wu D, et al. (2015) Graphene oxideBaGdF5 nanocomposites for multi-modal imaging and photothermal therapy. Biomaterials 42: 66-77.

25. Ou L, Bin Song, Huimin Liang, Jia Liu, Xiaoli Feng, et al. (2016) Toxicity of graphene-family nanoparticles: A general review of the origins and mechanisms. Particle and Fibre Toxicology 13.

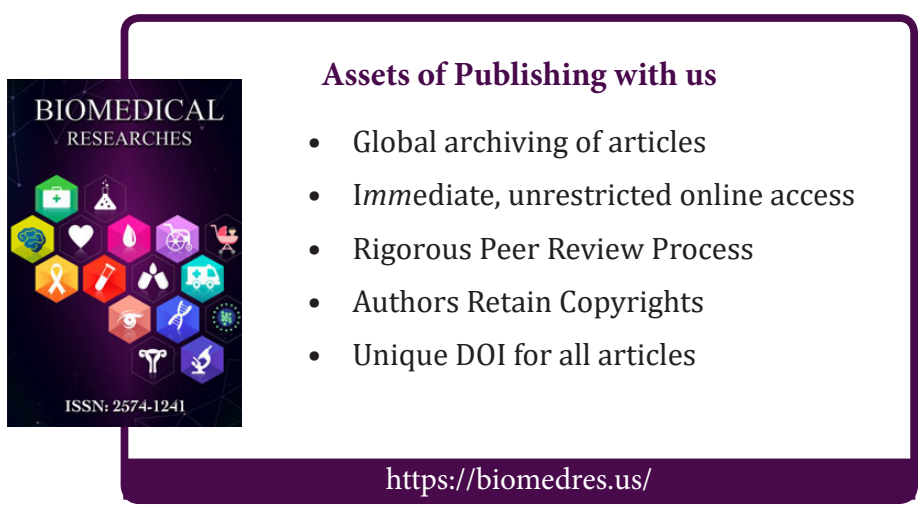

